\title{
Mouse Protein
}

National Cancer Institute

\section{Source}

National Cancer Institute. Mouse Protein. NCI Thesaurus. Code C26532.

Proteins found in any species or strain of mouse. 